\title{
P125 Local Pulse Wave Velocity in the Arterial Tree: Site Matters!
}

\author{
Daimé Campos Arias ${ }^{1,2,}$, Nikos Stergiopulos ${ }^{3}$, Tania Rodríguez Moliner $^{2}$, Patrick Segers ${ }^{1}$ \\ ${ }^{1}$ IBiTech-bioMMeda, Ghent University, Ghent, Belgium \\ ${ }^{2}$ BioMec, Cujae, Havana, Cuba \\ ${ }^{3}$ LHTC, EPFL, Lausanne, Switzerland
}

\section{ABSTRACT}

Background: Several so-called loop-based methods, have been proposed to estimate local pulse wave velocity (PWV). However, previous studies have demonstrated inaccuracies in local PWV-estimates due to presence of reflections, which led to the proposition of a frequency-domain correction method [1]. The aim of this study is to assess the accuracy of PWV-estimates from different loop methods throughout the human arterial tree.

Methods: The output data of a validated one-dimensional (1D) model of the human systemic circulation [2] was used to simulate the physiological signals needed on the estimations of local PWV methods, and this for model settings representing a young and an aged individual (stiffness increased by factor 2). Local PWV by the PU-loop, $\ln (\mathrm{D}) \mathrm{U}-\mathrm{loop}, \ln (\mathrm{D}) \mathrm{P}$-loop, QA-loop and the frequency-domain (PWV1-5) methods, were compared against the reference value obtained from the Bramwell-Hill equation.

Results: Figure 1 shows the deviation (\%) of loop-based estimates of PWV and PWV1-5 from the reference. The PU-loop overestimates PWV by more than $20 \%$ for most arterial sites, while the $\ln (\mathrm{D}) \mathrm{U}$ - and QA-loop underestimate to the same extent at these same locations. The correction method performs acceptably well in most of the young configuration. Discrepancies increase significantly in the aged model configuration for every studied method (except the $\ln (\mathrm{D}) \mathrm{P}$-loop method).

Conclusion: The accuracy of loop-based methods is highly dependent on the location where they are applied, and results should be interpreted with great caution. Best results were obtained for the reflection- insensitive $\ln (\mathrm{D}) \mathrm{P}$-loop method, but this method does not really provide an alternative for the Bramwell-Hill equation.

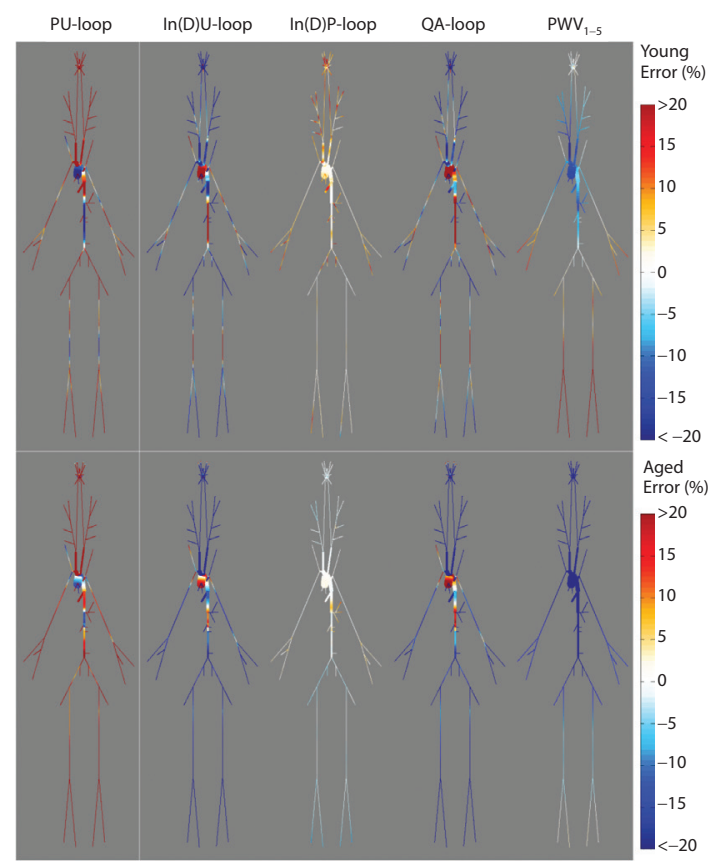

Figure 1 Deviation of the loop-based estimates of PWV (PU-, $\ln (\mathrm{D}) \mathrm{U}-, \ln (\mathrm{D}) \mathrm{P}$ - and QA-loops) and the correction method $\left(\mathrm{PWV}_{1-5}\right)$ from the reference (Bramwell-Hill), for every location of the arterial tree in the young and aged configurations. Red and blue colors indicate over- and underestimation, respectively. 


\section{REFERENCES}

[1] Segers P, Swillens A, Taelman L, Vierendeels J. Wave reflection leads to over- and underestimation of local wave speed by the PU- and QA-loop methods: theoretical basis and solution to the problem. Physiol Meas 2014;35:847-61.

[2] Reymond P, Merenda F, Perren F, Rüfenacht D, Stergiopulos N. Validation of a one-dimensional model of the systemic arterial tree. Am J Physiol Heart Circ Physiol 2009;297:H208-H22.

(c) 2019 Association for Research into Arterial Structure and Physiology. Publishing services by Atlantis Press International B.V. This is an open access article distributed under the CC BY-NC 4.0 license (http://creativecommons.org/licenses/by-nc/4.0/). 